\title{
Cifras estadísticas y políticas de salud pública que inciden en la mortalidad materna en gestantes entre las edades de 15 a 49 años en el departamento de Guainía comparado con el departamento de Antioquia en el periodo de 2018.
}

\author{
Sebastián Briceño Arias ${ }^{1 *}$, Paula Alejandra Cano Lizcanoํㅜ, María Camila Bocanegra Morenoํㅜ, Saida Garcia \\ Mayorca Madera ${ }^{1}$.
}

${ }^{1}$ Estudiantes de X semestre, facultad de Medicina, Fundación Universitaria Juan N. Corpas, Semillero de Salud Pública. Bogotá Colombia.

\section{RESUMEN}

Introducción: La elevada mortalidad materna en Colombia causa preocupación, por ello es importante abordar las estadísticas, causas, políticas de Salud Pública y determinantes en salud asociados a este fenómeno. Objetivo: Identificar las cifras estadísticas y políticas de Salud Pública que inciden en la mortalidad materna en las gestantes entre las edades de 15 a 49 años comparando los departamentos de Guainía y Antioquia en el 2018.

Metodología: Estudio observacional descriptivo de tipo ecológico. Incluyendo población gestante entre los 15 y 49 años de edad, de los departamentos estudiados. Se analizó información de fuentes estadísticas nacionales como el Departamento Nacional de Estadística (DANE) tabuladas en Excel 2.0, y bases de datos nacionales con publicaciones médicas y científicas. Se realizó una revisión bibliográfica de las políticas públicas en salud de los departamentos estudiados.

Resultados: En el año 2018 se presentó en el departamento de Guainía el pico más alto de defunciones maternas con una Razón de Mortalidad Materna (RMM) de 328.95 por cada 100.000 nacidos vivos. En el departamento de Antioquia, se evidencia un ligero aumento al año anterior con 48 defunciones y RMM de 64.98 por cada 100.000 nacidos vivos. Las principales causas en ambos departamentos fueron: trastornos hipertensivos en el embarazo, complicaciones del trabajo del parto y complicaciones en el puerperio. Las políticas de salud pública implementadas en Guainía cumplieron la meta de reducir a menos de 331.85 por cada 100.000 nacidos vivos la RMM, logrando 328.95 por cada 100.000 nacidos vivos en el 2018, pero enfocándose solo en planificación familiar, lo que ratifica la necesidad de tomar estrategias con las que cuenta Antioquia las cuales centran la salud pública en capacitación asistencial, control prenatal y transporte efectivo, manteniendo la reducción de la tendencia de sus cifras estadísticas en los últimos 4 años.

Conclusión: La RMM es menor en el departamento de Antioquia respecto al de Guainía, sin embargo, estos dos comparten las principales causas de mortalidad materna., lo que favorece la formulación de nuevas políticas públicas en Guainía siguiendo las realizadas en Antioquía que fueron más eficaces, apoyándose para este fin en los determinantes sociales particulares del departamento.

Palabras clave: Mortalidad materna, Determinantes sociales de la salud, Políticas públicas de salud, Causas de muerte, Embarazo.

\section{ABSTRACT}

Statistical data and public health policies that affect maternal mortality in pregnant women between the ages of 15 to 49 years in the department of Guainía compared with the department of Antioquia in the period of 2018.

Introduction: The high maternal mortality in Colombia causes concern, therefore it is important to address the statistics, causes, public health policies and health determinants associated with this phenomenon.
Objective: To identify the statistical figures and public health policies that affect maternal mortality in pregnant women between the ages of 15 to 49 years comparing the departments of Guainía and Antioquia in 2018.

Methodology: Ecological descriptive observational study. Including pregnant population between 15 and 49 years of age, from the departments of Guainía and Antioquia. Information from national statistical sources (DANE) tabulated in Excel 2.0, and national databases with medical and scientific publications were analyzed. A bibliographic review of the public health policies of the studied departments was carried out.

Results: In the year 2018, the highest peak of deaths was presented in the department of Guainía with a Maternal Mortality Ratio (MMR) of 328.95 per 100,000 live births. In the department of Antioquia, there is a slight increase from the previous year with 48 deaths and MMR of 64.98 per 100,000 live births. The main causes in both departments were: hypertensive disorders in pregnancy, complications of labor and complications in the puerperium. The public health policies implemented in Guainía met the goal of reducing the MMR to less than 331.85 per 100,000 live births, achieving 328.95 per 100,000 live births in 2018, but focusing only on family planning, which ratifies the need to take strategies such Antioquia that focus public health on care training, prenatal control and effective transportation, maintaining the reduction of the trend of their statistical figures in the last 4 years.

Conclusion: The MMR is lower in the department of Antioquia compared to that of Guainía, however, these two share the main causes of maternal mortality. Which favors the formulation of new public policies in Guainía following those carried out in Antioquia that were more effective, relying for this purpose on the particular social determinants of each department.

Keywords: Maternal mortality, Social determinants of health, Public health policy, Cause of death, Pregnancy.

\section{Autor de correspondencia: Briceño Arias Sebastián sebastian-briceno@juanncorpas.edu.co}

Como citar: Briceño Arias, S., Cano Lizcano, P., Bocanegra Moreno, M., \& Garcia Mayorca Madera, S. (2021). Cifras estadisticas y politicas de salud pública que inciden en la mortalidad materna en gestantes entre las edades de 15 a 49 años en el departamento de Guainía comparado con el departamento de Antioquia en el periodo de 2018. Revista Cuarzo, 27(1), 7-17.

Recibido: 11 de enero de 2021 Aceptado: 26 de febrero de 2021 Publicado: 30 de junio de 2021

Doi: https://doi.org/10.26752/cuarzo.v27.n1.523 


\section{INTRODUCCIÓN}

$\mathrm{L}$ a mortalidad materna es definida por la Organización Mundial de la Salud (OMS) como "la muerte de una mujer mientras está embarazada, dentro de los 42 días siguientes a la terminación del embarazo, independientemente de la duración y el sitio del embarazo, debida a cualquier causa relacionada con o agravada por el embarazo" (1), y es uno de los indicadores más sensibles del desarrollo de una nación debido a que sus niveles reflejan la equidad, el acceso a servicios de salud y obstétricos, entre otros aspectos. Es tan importante que fue incluida como uno de los objetivos de desarrollo sostenible relacionados con la salud: "Para el 2030, reducir la tasa mundial de mortalidad materna de 70 por cada 100.000 nacidos vivos" (2); pero esta meta no ha generado un cambio a simple vista si se consideran "las 830 mujeres que mueren diariamente en el mundo, de las cuales 21 fallecen en América del Sur, en su mayoría por causas prevenibles relacionadas con el embarazo, el parto y el postparto" (3). Lo anterior se ratifica con las cifras dadas por la Organización Panamericana de la Salud (OPS) con respecto a la situación de América del Sur, "el 75 por ciento de las muertes maternas en la región son causadas por hemorragias graves e infecciones, especialmente después del parto, hipertensión gestacional, preeclampsia y eclampsia" (3), lo cual lleva al planteamiento de cuáles son las causas que provocan que en el continente se tengan cifras tan alarmantes y qué medidas se están tomando para reducir estas cifras, "ya que está demostrado que el $99 \%$ de las defunciones maternas prevenibles ocurren en países de ingresos bajos y medianos, y esta cifra es aún mayor si entramos a comparar segmentos vulnerables de la sociedad" (3).

Cada día mueren aproximadamente 830 maternas por complicaciones obstétricas. En América Latina la razón de mortalidad materna promedio es de 270 por 100 mil nacidos vivos, resaltando que el $90 \%$ al $95 \%$ de las muertes maternas son evitables con una atención en salud oportuna y de calidad (4). Las principales causas de mortalidad materna a nivel mundial son: las hemorragias graves, las infecciones, la hipertensión inducida por el embarazo, las complicaciones en el parto y los abortos peligrosos (5).

Colombia es uno de los países que encabeza la lista con casos de mortalidad materna según las cifras del boletín epidemiológico del Instituto Nacional de Salud (INS) en la semana epidemiológica 52 de 2018, "la razón de mortalidad materna nacional preliminar era de 46,73 casos por 100.000 nacidos vivos" (6), mostrando otro panorama que permite ver que hay regiones del país y comunidades que bien no han sido parte de las estrategias del gobierno en cuanto a salud materna o tienen otros factores de riesgo y determinantes de la salud que hacen más susceptible a la población gestante de fallecer.

Por lo anterior, esta investigación busca conocer la situación de la mortalidad materna en mujeres de 15 a 49 años de edad en los departamentos de Guainía y Antioquia y comparar cifras, políticas de Salud Pública, estrategias que pudieran incidir en la misma en el periodo de 2018, buscando identificar las principales causas que conllevan a mortalidad materna en los departamentos estudiados y los posibles determinantes sociales en salud de las gestantes, para definir la manera en que estos se convierten en un factor de riesgo o protección que incide en la razón de mortalidad materna. Finalmente, se exponen las políticas de Salud Pública instituidas en cada departamento concerniente a la promoción y prevención de la mortalidad materna y si estas están siendo efectivas o no, realizando un análisis en ambos departamentos y su respectiva comparación.

\section{MATERIALES Y MÉTODOS}

Tipo y diseño del estudio: Este estudio es observacional descriptivo de tipo ecológico.

Población estudiada: Para esta investigación se incluye la población general de mujeres entre los 15 y 49 años que residen en el departamento de Guainía y en el departamento de Antioquia.

Criterios de selección: Se incluyó población de género femenino en condición de gestantes sin importar las semanas de gestación, con una edad entre los 15 y 49 años, residentes exclusivamente en el departamento de Guainía y/o Antioquia. Se excluyó a toda aquella población que no cumpliera con estos criterios.

Variables resultado: Razón de mortalidad materna, lugar de residencia, edad, prácticas culturales, cobertura sanitaria, distancia para acceder a servicios de salud, nivel de escolaridad, estrato socioeconómico y presencia de profesionales obstétricos.

Variables secundarias: Estado civil y empleo, raza, consultas de atención prenatal y consulta preconcepcional, cantidad de ambulancias y cantidad de camas.

Plan de recolección de datos: Para el plan de recolección de datos se comenzó planteando la pregunta de investigación basada en la problemática de Salud Pública respecto a la mortalidad materna, siendo esta: ¿Cuáles son las cifras estadísticas y políticas de salud pública que inciden en la mortalidad materna en las gestantes entre las edades de 15 a 49 años en el departamento de Guainía comparado con el departamento de Antioquia en el periodo de 2018? Conforme a esto, se consultaron las bases de datos de estadísticas vitales en el Departamento Administrativo Nacional de Estadística (DANE) para obtener la razón de mortalidad materna general en el departamento de Guainía y Antioquia por medio de los cuadros estadísticos de "defunciones maternas, por grupos de edad, según departamentos de residencia y grupos de causas de defunción (lista de causas agrupadas 6/67 Clasificación Internacional de Enfermedades-10 (CIE-10) de OPS" $(7,8)$ y así mismo los nacidos vivos en ese momento por medio de los cuadros de "Estadísticas vitales nacimientos y defunciones" específicamente en cuadros de "Nacimientos"(8), con una fecha de publicación que no sobrepasará los 5 años, con el fin de 
asegurar la información verídica más reciente y actualizada posible.

Con el fin de determinar las principales causas de mortalidad materna, se realizó una revisión bibliográfica el día 27 de septiembre de 2020 en el buscador Google Académico con la palabra clave "mortalidad materna" y se hallaron dos artículos: uno correspondiente a la Organización Mundial de la Salud siendo "Mortalidad materna" (5); y uno de la Secretaría Distrital de Salud de Bogotá, denominado de la misma manera (4). Luego de esto, se realizó una búsqueda en el DANE, de las principales causas de mortalidad materna en los departamentos de Guainía y Antioquia, mediante los cuadros estadísticos de "defunciones maternas, por grupos de edad, según departamentos de residencia y grupos de causas de defunción (lista de causas agrupadas 6/67 CIE-10 de OPS" $(7,8)$, con la información concerniente al año 2018.

Posteriormente, para abordar el tema de las políticas de Salud Pública implementadas en cada departamento en el año de estudio, el 2018, se recolectó información el 30 de septiembre en el buscador de Google Académico acerca de las generalidades en salud pública y su manejo en Colombia, abordando el departamento de Guainía en cuanto a políticas de salud propuestas para ese año, hallando un artículo denominado "INFORME EJECUTIVO DE GESTIÓN, VIGENCIA 2018"(9) de la Gobernación del departamento de la Guainía y una nota de la Secretaría de Salud Departamental llamada "GUAINÍA AVANZA EN LA PREVENCIÓN DE LA MORTALIDAD MATERNA" (10) todo esto para indagar las políticas implementadas a ese año.

Respecto a las políticas de Salud Pública presentes en el departamento de Antioquia, se evidencia que este departamento aplica y se rige por los "LINEAMIENTOS NACIONALES PARA LA REDUCCIÓN DE LA MORTALIDAD MATERNA"(11) que son una estrategia instaurada por el Ministerio de Protección Social, la cual establece una serie de parámetros para propender la maternidad segura; así mismo, la gobernación de Antioquia implementó la herramienta "MEMORIAS SOBRE VIGILANCIA EPIDEMIOLÓGICA DE MORTALIDAD MATERNA BASADA EN LA WEB"(12) con el propósito de identificar casos de mortalidad materna y así trabajar en conjunto con la Secretaria Seccional de Salud y Protección Social de Antioquia para realizar un acompañamiento del equipo de maternidad segura del Instituto Nacional de Salud. Verificando que todos los artículos fueran verídicos y de fuentes confiables, además cumpliendo con el criterio de tener una fecha de publicación menor a los últimos 5 años.

Finalmente, las condiciones en que nacen, crecen, viven y envejecen las personas fueron punto de comparación para los determinantes sociales en salud entre los departamentos de Guainía y Antioquia para establecer qué población tiene mayor riesgo de mortalidad materna. Estas circunstancias son el resultado de la distribución del poder, de las políticas, los recursos ambientales, el dinero, entre otros; y estas características ayudan a explicar las inequidades sanitarias y repercuten directamente en la salud de la población (13). Se realizó una búsqueda el 2 de octubre y se encontró un documento llamado "Análisis de la situación de salud según regiones en Colombia” (14), el cual comenta que gran parte del departamento es zona rural, por ende, es una región con altas tasas de enfermedades zoonóticas. En cuanto al departamento de Antioquia, se encontró un documento llamado "Análisis de situación de salud con el modelo de los determinantes sociales de salud" (15). En conjunto también se encontró mediante el Ministerio de Salud y Protección Social la cobertura de aseguramiento en salud respecto a los dos departamentos al año en estudio. Contando que todos los artículos fueran de fuentes confiables, además cumpliendo con el criterio de tener una fecha de publicación menor a los últimos 5 años.

Plan de análisis de datos: La información cuantitativa obtenida se unificó utilizando el programa informático Excel, presentándola en tabla y gráficas de razones de mortalidad en porcentaje finalizando con una descripción detallada de la situación asociada a la mortalidad materna. Luego, de manera independiente, se realizó la organización y análisis de los casos de mortalidad materna tanto en el departamento de Guainía como en el de Antioquia en el año 2018.

Tras la recolección de los datos sobre las causas más frecuentes de mortalidad materna en ambos departamentos, el análisis se realizó por medio de la comparación de estas causas de mortalidad materna entre los departamentos, y a su vez el paralelismo con las causas de mortalidad materna en América Latina y el mundo.

Para abordar el tema de políticas de salud en los departamentos de Guainía y Antioquia, se incluyó un contexto tanto general (a nivel nacional y mundial) como de cada departamento frente a las políticas de salud pública en conjunto con sus actividades y presupuestos. Luego, se hizo una comparación de los dos departamentos para saber si realmente las políticas implementadas fueron eficaces y redujeron la razón de mortalidad materna esperada para ese año.

Respecto a los determinantes sociales en salud en el departamento de Guainía de acuerdo a los documentos consultados, se prioriza que los factores ambientales y enfermedades transmitidas por vectores son los principales determinantes sociales en salud, así como cobertura de afiliación al SGSSS brindada por el Ministerio de Salud y Protección Social en el año de estudio (14). Para el departamento de Antioquia, se realizó un análisis sobre las circunstancias materiales de la población: condiciones de vida, disponibilidad de alimentos, condiciones de trabajo, factores conductuales, psicológicos y culturales, y el sistema sanitario, de igual forma, se hizo un análisis de los determinantes estructurales de las inequidades en salud: el estrato socioeconómico y el nivel educativo, la relación entre ellos y los efectos sobre la salud de la población del departamento, distrito y municipio (15).

Consideraciones éticas: Los autores declaran que para esta investigación no se han realizado experimentos con animales ni personas; además se han seguido los protocolos de publicación de datos de forma segura cumpliendo con los principios de 
privacidad. No se emplearon consentimientos informados debido a que no se trató información personal específica de alguna persona.

\section{RESULTADOS}

\section{A) Razón de Mortalidad Materna (RMM):}

En la gráfica y tabla 1, se muestra los resultados obtenidos de mortalidad materna en el departamento de Guainía, en el periodo 2015 a 2018 , encontrando que en el año 2015 no se presentaron defunciones sobre 587 nacidos vivos con una RMM de 0 ; frente al año 2016 con 2 defunciones sobre 641 nacidos vivos; dando RMM de 312,01 por cada 100.000 nacidos vivos. En el año 2017, se puede evidenciar que no se presentaron defunciones y el número de nacidos vivos aumentó a 833 con RMM de 0. En el 2018, que es el año de estudio, se obtuvo el pico más alto de defunciones con 3 defunciones y un número de nacidos vivos de 912 y la mayor RMM de 328,95 por cada 100.000 nacidos vivos.

\begin{tabular}{|c|c|c|c|}
\hline Años & $\mathbf{T D M}^{(1)}$ & $\mathbf{N V} \mathbf{V}^{(2)}$ & $\mathbf{R M M}^{(3)}$ \\
\hline 2015 & 0 & 587 & 0,00 \\
\hline 2016 & 2 & 641 & 312,01 \\
\hline 2017 & 0 & 833 & 0,00 \\
\hline 2018 & 3 & 912 & 328,95 \\
\hline
\end{tabular}

La gráfica de razón de mortalidad materna en el departamento de Guainía presenta tendencia al aumento, con el pico de mortalidad más notable en el año 2018.

Gráfica 1: Razón de mortalidad materna de Guainía (2015 - 2018).

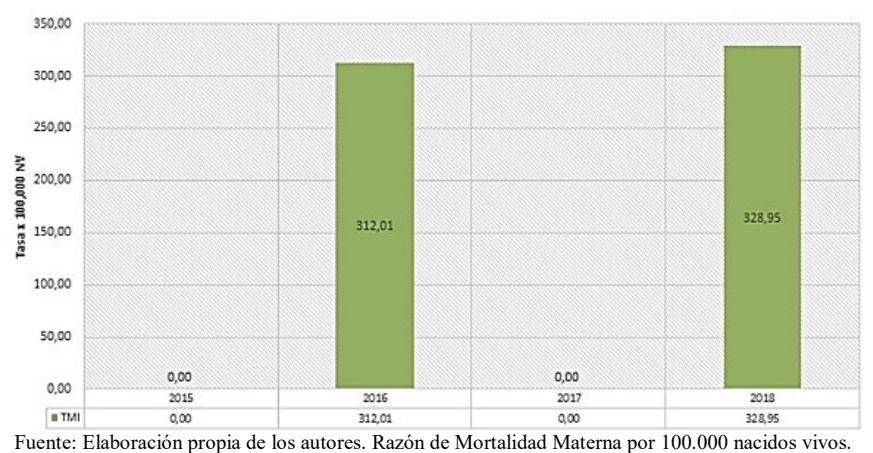

En la gráfica y tabla 2 de la mortalidad materna en el departamento de Antioquia, se tomaron datos entre los años 2015 a 2018. Se pudo identificar que en 2015 las defunciones maternas fueron 49 sobre 75.009 nacidos vivos con una RMM de 65,33 por cada 100.000 nacidos vivos, lo que aumenta con su pico más alto en el año 2016 con 53 defunciones sobre 74.197 nacidos vivos, con una RMM de 71,43 por cada 100.000 nacidos vivos. Aunque, en el año 2017 se observa la disminución más notoria de defunciones a 37 aumentando los nacidos vivos a 75.961, con una RMM de 48,71 por cada 100.000 nacidos vivos. En el 2018, se evidencia un pequeño aumento al año anterior con 48 defunciones y 73.866 nacidos vivos con una RMM de 64,98 por cada 100.000 nacidos vivos.

Tabla 2: Razón de mortalidad materna en función de defunciones maternas sobre nacidos vivos en el departamento de Antioquia.

\begin{tabular}{cccc}
\hline \hline Años & TDM(1) & NV(2) & RMM(3) \\
\hline \hline 2015 & 49 & 75.009 & 65,33 \\
2016 & 53 & 74.197 & 71,43 \\
2017 & 37 & 75.961 & 48,71 \\
2018 & 48 & 73.866 & 64,98 \\
\hline DANE.(1)TDM: Total de Defunciones Maternas; (2)NV: Nacidos Vivos; \\
(3)RMM: Razón de Mortalidad Materna por 100.000 nacidos vivos.
\end{tabular}

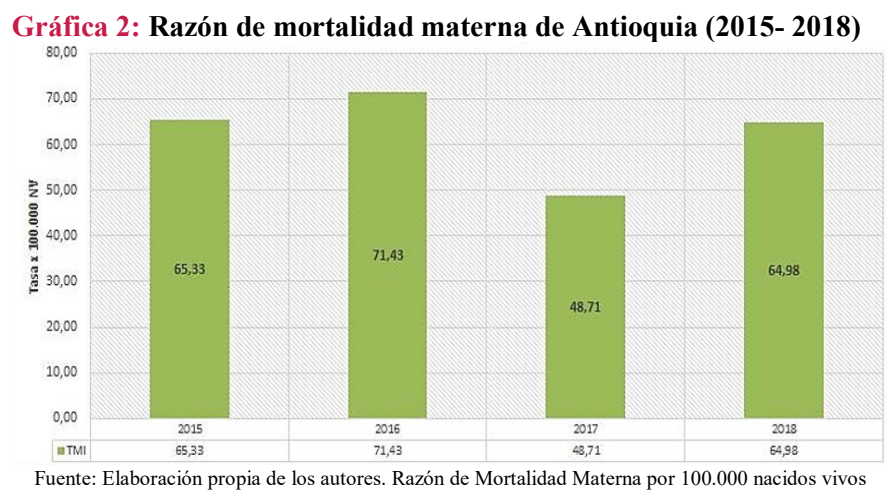

Respecto a la gráfica de razón de mortalidad materna en el departamento de Antioquia, muestra una tendencia a la disminución debido a que, en el 2018, aunque se produjo un pequeño aumento de casos de mortalidad, disminuyó frente a la tendencia general.

B) Principales causas de mortalidad materna en el departamento de Guainía comparadas a las del departamento de Antioquia:

Según cifras del DANE $(7,8)$, se presentó un caso de cada una de las siguientes causas de mortalidad materna para el departamento de Guainía en 2018 con su respectivo código CIE-10:

1. Edema, proteinuria y trastornos hipertensivos en el embarazo, el parto y el puerperio (O10-O16).

2. Complicaciones del trabajo del parto y del parto (O60O75).

3. Complicaciones principalmente relacionadas con el puerperio (O85-O92).

Respecto a las cifras mostradas, se precede que en el 2018 las tres principales causas encontradas tuvieron el mismo porcentaje de incidencia en el departamento, generando una gran interrogante sobre el por qué se produjeron estas muertes y si las políticas públicas están siendo efectivas. 
Por otro lado, en cuanto a las principales causas de mortalidad materna con su respectivo código CIE-10 para el departamento de Antioquia, en el año 2018 fueron $(7,8)$ :

1. Otras afecciones obstétricas no clasificadas en otra parte (O95-099), esta clasificación corresponde al $65,3 \%$ de la totalidad de muertes maternas en este departamento.

2. Edema, proteinuria y trastornos hipertensivos en el embarazo, el parto y el puerperio (O10-O16), corresponde al 12,2\%.

3. Complicaciones del trabajo del parto y del parto (O60O75), corresponde al 6,12\%.

4. Complicaciones principalmente relacionadas con el puerperio (O85-O92), corresponde al 6,12\%.

El departamento de Antioquia, en el 2018 se encuentra que la causa de "Otras afecciones obstétricas no clasificadas en otra parte (O95-O99)" fue la más alta siendo un 65,3\% por el total de las muertes maternas, y en segundo lugar "Edema, proteinuria y trastornos hipertensivos en el embarazo, el parto y el puerperio (O10-O16)" con un 12,2\%, siendo ambas las causales más importantes. Teniendo en cuenta que en Antioquia se presenta un mayor número de mortalidades maternas, genera gran inquietud si hay un manejo adecuado de la promoción, prevención y seguimiento de la gestación, atención del parto y puerperio en las mujeres del departamento.

En cuanto a la comparación de las causas de mortalidad materna en ambos departamentos, puede observarse una similitud en tres causas principales que se presentan simultáneamente en estos, como lo son "Edema, proteinuria y trastornos hipertensivos en el embarazo, el parto y el puerperio (O10-O16)", "Complicaciones del trabajo del parto y del parto (O60-O75)" y "Complicaciones principalmente relacionadas con el puerperio (O85-O92)", y que a su vez se relacionan con las causas a nivel mundial (a excepción del aborto inseguro). Se destaca que solo en el departamento de Antioquia se presenta la etiología "Otras afecciones obstétricas no clasificadas en otra parte (095-099)", ya que al no tener claridad respecto a las patologías específicas que engloba esta etiología, no puede darse un tratamiento diferencial en políticas de salud que intervengan en la aparición de esta, la complicación que con mayor frecuencia provoca muertes maternas en ese departamento.

Es importante mencionar algunos determinantes sociales que se relacionan con la mortalidad materna por las causas anteriormente mencionadas: la pobreza, la distancia, la falta de información, la inexistencia de servicios adecuados y las prácticas culturales (5); estos factores explican el hecho de que la mortalidad materna es mayor en países de ingresos bajos y que pueden ser la base para desarrollar políticas en salud dirigidas a salvaguardar la vida de las maternas.

\section{C) Políticas de salud pública tomadas por cada departamento frente a la promoción y prevención de la mortalidad materna:}

Respecto al departamento de Guainía por medio del "Informe Ejecutivo de Gestión” (9), publicado por la Gobernación del departamento de Guainía en el año 2018, se expresó todas las acciones orientadas de acuerdo al plan de desarrollo "Vamos Pa Lante Guainía 2016-2019", en este se analizó todos los planes de políticas en Salud Pública, los cuales se expresarán a continuación.

Antes de plantear todas las estrategias de salud pública gestionadas en el año en estudio, se hablará un poco de cómo se clasificó la mortalidad materna en torno al informe de la Gobernación de Guainía.

\section{Se clasificó dentro del "Subprograma: Dimensión} Sexualidad, Derechos Sexuales y Reproductivo" (9) en donde se manejaban los indicadores de Mortalidad Materna y el de porcentaje de adolescentes (12 - 18 años) en estado de embarazo en el departamento. Iniciando las actividades de políticas de Salud Pública con una línea de base de Razón de Mortalidad Materna de 179.5 x 100,000 Nacidos Vivos (NV) con un objetivo de reducir la razón de mortalidad materna a 47 por cada $100,000 \mathrm{NV}$ a nivel nacional, con una meta departamental para el 2018 de aproximadamente 331.85 x 100,000 NV.

Las actividades presentadas en este año para reducir la mortalidad materna se presentan en la tabla 3.

Tabla 3: Actividades gestionadas en el año 2018 por parte del departamento de Guainía conforme a las políticas de Salud Pública ACTIVIDADES DESCRIPCIÓN

Vigilancia en salud pública en los Se implementó la estrategia "Planifica tu eventos relacionados con el familia", logrando educar en planificación programa de salud sexual y familiar a 682 mujeres en edad fértil y el reproductiva, para realizar el BAI, ingreso al programa de planificación familiar visitas de campo, búsquedas a 140 mujeres donde la Institución Prestadora comunitarias y estudios de Salud (IPS) administro métodos epidemiológicos: hormonales.

Con el apoyo de la OPS, se realizó entrega a la IPS Nuevo Hospital Manuel Elkin Patarroyo ubicado en Inírida, Guainía; de 4 kits de parto y 4 trajes anti choque, capacitando a 25 trabajadores de salud (Médicos y Ginecobstetras) de la IPS en el manejo de estos kits.

Se brindó atención integral a pacientes, promoción de la salud y prevención de enfermedades en la población damnificada a nivel socioeconómico del departamento de Guainía.

Canalización de Gestantes 22, en planificación familiar 95, desparasitación en 421 menores de 10 años.

"Informe Ejecutivo de Gestión”, Gobernación del departamento de Guainía. 2018(9).

El presupuesto manejado para este subprograma fue de $\$ 159.057 .789$ representando el $63.60 \%$ del presupuesto total del informe ejecutivo de gestión para el departamento de Guainía.

Por otro lado, en el año 2018 la Gobernación de este departamento junto con la Organización Panamericana de la Salud a través de FECOLSOG (Federación Colombiana de Obstetricia y Ginecología) y en coordinación con la Secretaría de Salud Departamental y la dimensión Sexualidad Derechos Sexuales y Reproductivos(10) realizaron múltiples actividades con el objetivo de reducir la mortalidad materna en este lugar, debido a su importancia como evento en salud pública, y 
fomentar la atención en salud de calidad para esta población, entre las que se encuentra la vigilancia a los procesos de atención en la IPS Nuevo Hospital Manuel Elkin Patarroyo a las gestantes del departamento; Por otro lado, en el año 2018 la Gobernación de este departamento junto con la Organización Panamericana de la Salud a través de FECOLSOG (Federación Colombiana de Obstetricia y Ginecología) y en coordinación con la Secretaría de Salud Departamental y la dimensión de Sexualidad Derechos Sexuales y Reproductivos(10) realizaron múltiples actividades con el objetivo de reducir la mortalidad materna en este lugar debido a su importancia como evento en salud pública, además de fomentar la atención en salud de calidad para esta población, entre las que se encuentra la vigilancia a los procesos de atención en la IPS Nuevo Hospital Manuel Elkin Patarroyo a las gestantes del departamento; en esta IPS se realizó la ejecución de un taller sobre manejo de la hemorragia posparto, trastornos hipertensivos y manejo del traje anti choque para el personal de salud (médicos y ginecobstetras) de la IPS, con posterior entrega de cuatro kits de emergencia obstétrica, cuatro kits de parto y cuatro trajes anti choque al departamento por parte de la OPS en el primer semestre del 2018. Dando así el cumplimiento a las acciones efectuadas para evitar la muerte de las maternas dentro del contexto de atención en salud en Guainía (10).

Imagen 1: Trajes anti-choque entregados en Guainía por la OPS. Tomado de: Gobernación del Guainía y Secretaría de Salud Departamental

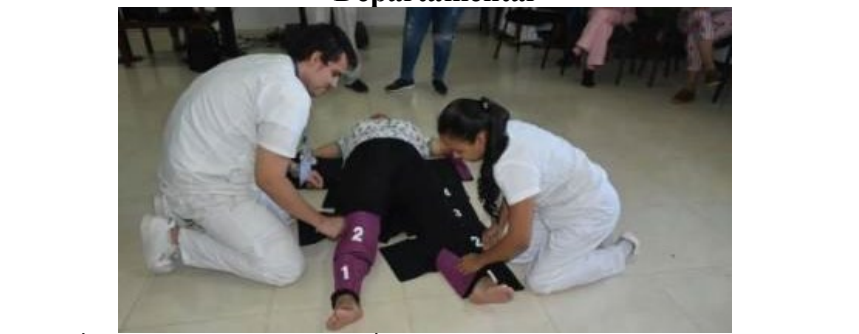

GUAINÍA AVANZA EN LA PREVENCIÓN DE LA MORTALIDAD MATERNA [Imagen]. Nota: 024. Guainía: Gobernación del Guainía y Secretaría de Salud Departamental; 2018

Respecto a las políticas de Salud Pública establecidas en Antioquia, la Gobernación de este departamento sigue los lineamientos nacionales vigentes para la reducción de la mortalidad materna en el 2010(11) y están estipulados en una carta circular del Ministerio de Salud y Protección Social que a su vez realiza seguimiento trimestral que rige a todo el departamento, en dicho documento partir del apartado 5, describen los siguientes aspectos sobre mortalidad materna:

5. Se pretender asegurar Atención Obstétrica Ambulatoria y de Emergencia en todos los sitios donde las mujeres viven durante su embarazo y donde se lleva a cabo el parto, lo anteriormente estipulado implica el desarrollo de procesos de auditoría que permitan garantizar en todas las instituciones los elementos e insumos críticos necesarios para resolver la emergencia obstétrica, para esto se sugiere que con el apoyo de la Oficina de Inspección Vigilancia y Control se verifique el cumplimiento de indicadores de la OMS por tanto, se propone un curso para revisar los alcances que han desarrollado los hospitales Instituciones Amigas de la Mujer y la Infancia (IAMI) en el cumplimiento de "atender con prácticas seguras el parto y el puerperio" para dar adecuada atención a las necesidades de las mujeres(10).

6. Garantizar que las personas encargadas de realizar el control prenatal cuenten con el entrenamiento requerido para detectar precozmente las alteraciones durante el embarazo y sobre todo, estabilizar y remitir de la manera más segura a las mujeres gestantes en las que se presente una emergencia obstétrica. Es importante enfatizar en el hecho de que a toda materna se le debe evaluar el riesgo obstétrico en cada contacto con los servicios de salud (sea este promotor/a, auxiliar, o profesional de enfermería o medicina) (10).

7. Propender que en todas las salas de trabajo de parto, parto y puerperio haya profesionales capacitados en la asistencia de la labor del parto, y en el correcto manejo y atención de las complicaciones relacionadas con el evento obstétrico en este periodo (10).

8. Garantizar que en las salas de puerperio exista el personal médico y de enfermería en número suficiente $y$ entrenado en la detección y manejo de las complicaciones del puerperio, con el fin de reducir la morbilidad y mortalidad evitables que con mayor frecuencia ocurren en este período, como son la hemorragia en el posparto inmediato y las infecciones (10).

9. Asegurar la comunicación y el transporte efectivo desde todos los sitios donde estén las gestantes mediante el establecimiento de alianzas con la comunidad para que toda mujer embarazada tenga definido el medio de transporte que la llevará desde su casa al hospital o centro de atención más cercano, o donde se le programe la atención de su parto (10).

10. En el tema de garantía de derechos a las mujeres y hombres, queda claro que las Entidades Prestadoras de Salud (EPS) deben comprometerse a desarrollar acciones de orientación a la demanda temprana de servicios en salud materna (10).

11. Comprometer a aseguradoras y prestadores en la necesidad de dar un trato de urgencia al manejo de las referencias de las gestantes. De igual manera, enviar contra referencia no solo a la central de costos de las Secretarías de Salud Departamentales, sino a la institución que referencia en primera instancia a la materna (10).

13. Recordar y garantizar que todo contacto con mujeres en edad fértil es una oportunidad para tomar una citología, hacer una serología, informar sobre el acceso a servicios básicos en SSR, direccionar a consejería en SSR, o específicamente en anticoncepción y VIH (10).

14. Realizar un plan que permita el seguimiento a la calidad de la atención materna y perinatal a través de la auditoría de historias clínicas, de servicios de control prenatal o de atención institucional del trabajo de parto. 
15. Realizar auditoria de todos los procesos de atención en acciones de detección precoz y atención oportuna en presencia de daños y lesiones en Salud Sexual y Reproductiva (10).

16. Cumplir con los compromisos establecidos durante las reuniones regionales realizadas con el Instituto Nacional de Salud y la Superintendencia (10).

La Secretaría Seccional de Salud y Protección Social de Antioquia, presentó las Memorias sobre vigilancia epidemiológica de mortalidad materna basada en la Web (12), con el acompañamiento del equipo de Maternidad Segura del Instituto Nacional de Salud, realiza jornadas de asesoría y asistencia técnica sobre vigilancia de la mortalidad materna basada en la Web, dirigidas a los responsables de la vigilancia y de la aplicación del Sistema de Vigilancia en Salud Pública (SIVIGILA) (12)

Haciendo una comparación y análisis en los departamentos de Guainía y Antioquia conforme a las políticas de Salud Pública para el 2018, se puede afirmar que el departamento de Antioquia tuvo un programa de políticas de Salud Pública mejor estructurado y completo, incluyó asegurar la Atención Obstétrica Ambulatoria y de Emergencia en todo el departamento, garantizando los insumos necesarios para la atención del parto, cumpliendo con la capacitación del personal de salud en la atención del control prenatal, entrenamiento para asistir y acompañar la labor del parto al personal de salud que labora en salas de parto para detección precoz y correcto manejo de las complicaciones puerperales, asegurar comunicación y transporte efectivo de manera terrestre, desde todos los sitios donde estén las gestantes, mediante el establecimiento de alianzas con la comunidad, efectivo plan de seguimiento a la calidad de la atención materna y perinatal a través de la auditoría de historias clínicas de servicios de Control Prenatal o de Atención Institucional del trabajo de parto, política que consideramos de suma importancia (11).

De acuerdo a lo anterior, a pesar de la escasa planeación de políticas de salud pública en el departamento de Guainía en 2018 la meta de reducir la razón de mortalidad materna, cumpliendo con la meta sobre el límite el cual era aproximadamente $331.85 \times 100,000 \mathrm{NV}$, con una RMM de 328.95. En comparación, el departamento de Antioquia, al tener un plan de Salud Pública mucho más enfocado y completo, las cifras de mortalidad materna en los últimos 4 años presentaron tendencia al decremento, lo que quiere decir que tanto el departamento de Guainía como el de Antioquia deben mejorar la implementación de esas actividades y políticas que ayuden a seguir reduciendo sus razones de mortalidad en los próximos años.

\section{D) Determinantes sociales en salud de las gestantes del departamento de Guainía comparados con las del departamento de Antioquia:}

En el departamento de Guainía se determinó que la contaminación ambiental por mercurio utilizado para la minería, la etnicidad, la desnutrición, el bajo nivel socioeconómico, la lejanía a servicios de salud de nivel superior y las inundaciones en ola invernal, son los principales determinantes sociales en salud del departamento(14), la mayor parte de su extensión es zona rural, por ende, es una región con altas tasas de enfermedades zoonóticas, transmitidas por vectores en su mayoría (malaria, dengue, leishmaniasis, enfermedad de Chagas y fiebre amarilla). De igual forma, la mayoría de la población cuenta con régimen subsidiado (14).

Según el Ministerio de Salud y Protección Social (16), al año 2018 el departamento de Guainía cuenta con una cobertura de aseguramiento en salud ilustrada en la tabla 4.

\begin{tabular}{|c|c|c|c|}
\hline & Nacional & $\begin{array}{c}\text { Departamento } \\
\text { Guainía }\end{array}$ & $\begin{array}{l}\text { Municipio } \\
\text { Todo }\end{array}$ \\
\hline & Diciembre 2018 & Diciembre 2018 & Diciembre 2018 \\
\hline Sisbén 1 & 27.140 .038 & 8.224 & 8.224 \\
\hline Sisbén 2 & 2.787 .974 & 1.247 & 1.247 \\
\hline PPNA & 525.698 & 289 & 289 \\
\hline Contributivo & 22.378 .384 & 5.268 & 5.268 \\
\hline Subsidiado & 22.658 .108 & 38.166 & 38.166 \\
\hline $\begin{array}{l}\text { Excepción \& } \\
\text { Especiales }\end{array}$ & 2.138 .117 & 880 & 880 \\
\hline Afiliados & 47.174.609 & 44.314 & 44.314 \\
\hline Población DANE & 49.834 .240 & 43.446 & 43.446 \\
\hline Corbertura & $94,66 \%$ & $100,00 \%$ & $100,00 \%$ \\
\hline
\end{tabular}

Fuente: Ministerio de Salud y Protección Social. Protección social. Cifras de aseguramiento en salud. Departamento de Guainía [Tabla]. Cifras de aseguramiento en salud; 2018. SISBEN: Sistema de Identificación y Clasificación de Potenciales Beneficiarios para Programas Sociales. DANE: Departamento Administrativo Nacional de Estadística.

En la tabla 4 se muestra que en el departamento hay un aseguramiento en salud respecto a SISBEN 1 de 8,224 afiliados y SISBEN 2 de 1,247, con un aporte al régimen subsidiado de 38,166 y contributivo de 5,268 afiliados, exhibiendo un total de afiliados de 44,314 con una cobertura del $100 \%$.

En el departamento de Antioquia en el año 2015, el $97.65 \%$ de la población contaba con servicios de electricidad en el área rural y el $100 \%$ de la población tenía este mismo servicio en el área urbana; el $95.47 \%$ de los habitantes tenía servicios de acueducto y el $94.7 \%$ servicio de alcantarillado, el $5.08 \%$ de la población general en el departamento presenta dificultad para disponer de los alimentos. El porcentaje de nacidos vivos con bajo peso al nacer en el departamento, muestra para los años 2009,2012 y 2015 una tendencia al decremento (16). En lo que concierne a las condiciones de trabajo, no hay datos oficiales sobre el tipo de economía (informal o formal), de igual forma no existen reportes de enfermedades laborales. Referente a los factores psicológicos y culturales, la violencia intrafamiliar es baja para el año 2016 con una tasa de 15.85 casos por 1,000 habitantes; la violencia contra la mujer en el mismo año presentó una tasa de 119 casos por 1,000 habitantes (15).

Los indicadores del sistema sanitario, para el año 2016, las coberturas de vacunación contra la tuberculosis para nacidos vivos, muestra una tendencia al incremento, la cobertura de parto institucional y el porcentaje de partos atendidos por 
personal calificado, cuenta con una cobertura del $100 \%$. En educación en el año 2016, la tasa de cobertura de educación primaria fue del $89.45 \%$, la tasa de cobertura de educación secundaria fue del $92.05 \%$ y la tasa de cobertura de educación media fue del $70.46 \%$ (15).

Según el Ministerio de Salud y Protección Social (16), al año 2018 el departamento de Antioquia cuenta con una cobertura de aseguramiento en salud ilustrada en la tabla 5.

\begin{tabular}{|c|c|c|c|}
\hline & Nacional & $\begin{array}{l}\text { Departamento } \\
\text { Antioquia }\end{array}$ & $\begin{array}{l}\text { Municipio } \\
\text { Todo }\end{array}$ \\
\hline & Diciembre 2018 & Diciembre 2018 & Diciembre 2018 \\
\hline Sisbén 1 & 27.140 .038 & 3.116 .603 & 3.116 .603 \\
\hline Sisbén 2 & 2.787 .974 & 639.060 & 639.060 \\
\hline PPNA & 525.698 & 41.641 & 41.641 \\
\hline Contributivo & 22.378 .384 & 3.821 .627 & 3.821 .627 \\
\hline Subsidiado & 22.658 .108 & 2.338 .345 & 2.338 .345 \\
\hline $\begin{array}{c}\text { Excepción \& } \\
\text { Especiales }\end{array}$ & 2.138 .117 & 104.367 & 104.367 \\
\hline Afiliados & 47.174 .609 & 6.264 .339 & 6.264 .339 \\
\hline Población DANE & 49.834 .240 & 6.691 .030 & 6.691 .030 \\
\hline Corbertura & $94,66 \%$ & $93,62 \%$ & $93.63 \%$ \\
\hline
\end{tabular}

Fuente: Ministerio de Salud y Protección Social. Protección social. Cifras de aseguramiento en salud. Departamento de Antioquia [Tabla]. Cifras de aseguramiento en salud; 2018. SISBEN: Sistema de Identificación y Clasificación de Potenciales Beneficiarios para Programas Sociales. DANE: Departamento Administrativo Nacional de Estadística.

La tabla 5 muestra que en el departamento hay un aseguramiento en salud respecto a SISBEN 1 de 3,116,603 afiliados y SISBEN 2 de 639,060; con un aporte al régimen subsidiado de 2,338,345 y contributivo de 3,821,627 afiliados, mostrando un total de afiliados de 6,264,339 con una cobertura del $93.62 \%$.

La tabla 6 define los determinantes sociales en salud que inciden en la mortalidad materna de ambos departamentos según los hallazgos encontrados:

Tabla 6: Comparación de determinantes sociales entre el departamento de Guainía y el departamento de Antioquia.

\begin{tabular}{lll}
\hline \hline \multicolumn{2}{c}{ Determinantes sociales en salud que inciden en la MM } \\
\hline $\begin{array}{c}\text { Determinantes de la } \\
\text { Salud }\end{array}$ & \multicolumn{1}{c}{ Guainía } & \multicolumn{1}{c}{ Antioquia } \\
\hline \hline Cobertura electricidad & $\begin{array}{l}\text { Sí, pero no en todo el } \\
\text { territorio. }\end{array}$ & $\begin{array}{l}\text { Cobertura del 97.65\% de la } \\
\text { población en el área rural, y } \\
100 \% \text { de la población en el } \\
\text { área urbana. }\end{array}$ \\
\hline Cobertura acueducto & $\begin{array}{l}\text { Sí, pero no en todo el } \\
\text { territorio. }\end{array}$ & $\begin{array}{l}\text { Cobertura del 95.47 \% de la } \\
\text { población. }\end{array}$ \\
\hline $\begin{array}{l}\text { Cobertura } \\
\text { alcantarillado }\end{array}$ & $\begin{array}{l}\text { Sí, pero no en todo el } \\
\text { territorio. }\end{array}$ & $\begin{array}{l}\text { Cobertura del 94.7\% de la } \\
\text { población. }\end{array}$ \\
\hline Desnutrición & $\begin{array}{l}\text { Sí, en la población } \\
\text { indígena. }\end{array}$ & $\begin{array}{l}5.08 \% \text { de la población tiene } \\
\text { dificultades al momento de } \\
\text { acceder al alimento. }\end{array}$ \\
\hline $\begin{array}{l}\text { Incidencia violencia } \\
\text { familiar }\end{array}$ & No se encuentran datos. & $\begin{array}{l}\text { Tasa de 15,85 casos por 1000 } \\
\text { habitantes. }\end{array}$ \\
\hline $\begin{array}{l}\text { Incidencia violencia } \\
\text { contra la mujer }\end{array}$ & No se encuentran datos. & $\begin{array}{l}\text { Tasa de 119 casos por 1000 } \\
\text { habitantes. }\end{array}$ \\
\hline & $\begin{array}{l}\text { La mayoría de la } \\
\text { población tiene } \\
\text { régimen subsidiado. } \\
\text { Cobertura de afiliación } \\
\text { al SGSSS }\end{array}$ & $\begin{array}{l}\text { Cobertura del 93,8 \% de la } \\
\text { población. }\end{array}$ \\
100\% de la población. & \\
\hline
\end{tabular}

\begin{tabular}{|c|c|c|}
\hline $\begin{array}{l}\text { Cobertura vacunación } \\
\text { con BCG }\end{array}$ & No se encuentran datos. & Tendencia al incremento. \\
\hline $\begin{array}{l}\text { Cobertura parto } \\
\text { institucional }\end{array}$ & $\begin{array}{l}\text { Partos atendidos por } \\
\text { parteras. }\end{array}$ & Cobertura $100 \%$. \\
\hline $\begin{array}{l}\text { Partos atendidos por } \\
\text { personal calificado }\end{array}$ & $\begin{array}{l}\text { Partos atendidos por } \\
\text { parteras. }\end{array}$ & Cobertura $100 \%$. \\
\hline $\begin{array}{l}\text { Cobertura de educación } \\
\text { primaria }\end{array}$ & No se encuentran datos. & $\begin{array}{l}\text { Cobertura de } 89.45 \% \text { de la } \\
\text { población. }\end{array}$ \\
\hline $\begin{array}{l}\text { Cobertura de educación } \\
\text { secundaria }\end{array}$ & No se encuentran datos. & $\begin{array}{l}\text { Cobertura de } 92.05 \% \text { de la } \\
\text { población. }\end{array}$ \\
\hline $\begin{array}{l}\text { Cobertura de educación } \\
\text { media }\end{array}$ & No se encuentran datos. & $\begin{array}{l}\text { Cobertura de } 70,46 \% \text { de la } \\
\text { población. }\end{array}$ \\
\hline Nivel socioeconómico & Bajo - Medio. & Medio - Alto. \\
\hline $\begin{array}{c}\text { Determinantes de la } \\
\text { Salud }\end{array}$ & Guainía & Antioquia \\
\hline Población étnica & Sí. & No. \\
\hline $\begin{array}{l}\text { Lejanía a servicios de } \\
\text { salud de mayor nivel }\end{array}$ & Sí. & No. \\
\hline Ruralidad & $\begin{array}{l}\text { - Contaminación } \\
\text { ambiental por mercurio } \\
\text { utilizado para la } \\
\text { minería. } \\
\text { - Inundaciones en ola } \\
\text { invernal. } \\
\text { - Altas tasas de } \\
\text { enfermedades } \\
\text { zoonóticas: malaria, } \\
\text { dengue, leishmaniasis, } \\
\text { enfermedad de Chagas, } \\
\text { fiebre amarilla. }\end{array}$ & $\begin{array}{l}\text { - El } 33,90 \% \text { de la población } \\
\text { no tiene acceso a fuentes de } \\
\text { agua mejorada. } \\
\text { - El } 28,33 \% \text { de la población } \\
\text { tiene inadecuada eliminación } \\
\text { de excretas. }\end{array}$ \\
\hline $\begin{array}{l}\text { Enfermedad } \\
\text { periodontal }\end{array}$ & $\begin{array}{l}\text { Gingivitis y } \\
\text { periodontitis. }\end{array}$ & No se encuentran datos. \\
\hline
\end{tabular}

\section{DISCUSIÓN}

El presente estudio de acuerdo con los resultados obtenidos mediante cuadros estadísticos sobre mortalidad materna, causas principales de mortalidad, políticas de salud pública y determinantes sociales, permite comparar y analizar con el estudio hecho por Sandoval y Eslava acerca de las "Inequidades en mortalidad materna por departamentos en Colombia para los años (2000-2001), (2005-2006) y (2008-2009)"(17), encontrándose lo siguiente:

Al calcular las Razones de Mortalidad Materna Acumulada Estandarizada (RMMAe) para los períodos 2000-2001, 20052006 y 2008-2009, se obtuvieron cifras de 81 y 161 por 100,000 nacidos vivos. Entre los departamentos que registraron las razones de mortalidad materna más alta se encontró el territorio de Guainía, en el periodo 2005-2006, presentando una razón de 100 por 100,000 nacidos vivos.

Por otro lado, se determinó que, para los periodos evaluados, las tres principales causas de mortalidad materna en Colombia fueron los mismos: "trastornos hipertensivos en el embarazo; otras condiciones obstétricas no clasificadas en otra parte y complicaciones del trabajo del parto y del parto"(17), predominando la etiología de edema y trastornos hipertensivos en el embarazo para el periodo 2000-2001, y otras afecciones obstétricas no clasificadas en otra parte en los periodos 2005 2006 y 2008-2009.

Como objetivo se planteó la búsqueda de aquellas inequidades en salud que constituyen determinantes sociales que pueden incidir en un desenlace negativo en Salud Pública 
como la mortalidad materna en el país, evidenciando que con el paso de los años se ha aumentado esa brecha de acceso a la salud en Colombia al calcular la Fracción Atribuible (que mide el riesgo que tiene una gestante de morir por el solo hecho de vivir en Colombia, y no en los países usados como referentes en cada año), con resultados que revelan un exceso de riesgo del $82 \%$ para el periodo $2000-2001$, de $88 \%$ para el periodo $2005-2006$ y $87 \%$ para el periodo $2008-2009(17)$.

Departamentos como Guainía se encontraron en exceso de riesgo entre 83 y $95 \%$, mientras el departamento de Antioquia se encontró entre 60 y $71 \%$, lo que se relaciona con los datos analizados de población con necesidades básicas insatisfechas (NBI) en el año 2005, puesto que departamentos como Guainía cuentan con mayor proporción de población con NBI de $60,6 \%$, lo que explica la Fracción Atribuible tan alta ya nombrada; mientras que el departamento de Antioquia presenta una proporción de población con NBI menor al territorio de Guainía $22,9 \%$, lo que a su vez puede vincularse con la Fracción Atribuible menor a ese departamento.

Se identificó la inequidad en la mortalidad materna y la proporción de población con NBI, lo que pretende ser una guía para describir los determinantes de salud que componen a las diferentes regiones colombianas y que puedan ser usados como fundamento en la generación de políticas de salud pública que pretendan reducir este fenómeno de importancia mundial. En la tabla 7 se observa una comparación de los resultados obtenidos en esta investigación respecto a un trabajo similar.

Las cifras de mortalidad materna para el departamento de Guainía durante las últimas décadas han sido de gran alarma tanto para el mismo departamento como para el Gobierno Nacional, debido a que cada año viene con un alza en sus razones de mortalidad materna siendo al año 2018 el departamento con mayor razón de mortalidad materna (7).

Durante casi dos décadas las causas principales de mortalidad materna son: "edema, proteinuria y trastornos hipertensivos en el embarazo, el parto y el puerperio (O10-O16)" y "Complicaciones del trabajo del parto y del parto (O60-O75)". En estas se podrían buscar en los próximos años estrategias o políticas de salud pública para reducir o controlar estas causalidades de mortalidad en las gestantes colombianas, respectivamente del departamento de Guainía y Antioquia.

Referente a los determinantes sociales incidentes en los departamentos en Colombia para mortalidad materna se sigue ratificando que el departamento de Guainía cuenta con uno de las mayores proporciones de NBI (Necesidades Básicas Insatisfechas), así mismo cuenta con determinantes negativos como lo son: el riesgo a padecer de enfermedades como malaria, dengue, leishmaniasis, enfermedad de Chagas y fiebre amarilla; además de ubicarse a grandes distancias de los centros de salud con niveles altos de atención. También cabe resaltar que en este departamento gran parte de los partos son guiados por partera y un alto número de esta población está afiliada a régimen subsidiado lo cual quiere decir que son servicios brindados por el estado debido a su nivel socioeconómico bajo. Respecto a
Antioquia, se determina que tiene una proporción de NBI estándar, contando más específicamente con: la atención de partos por profesionales capacitados y en centros médicos, niveles económicos superiores, viviendas ubicadas principalmente en áreas urbanas, con mejor acceso a centros de salud, y tienen mayores niveles educativos que las gestantes del departamento de Guainía.

Tabla 7: Comparación de los resultados obtenidos en el trabajo "Inequidades en mortalidad materna por departamentos en Colombia para los años (2000-2001), (2005-2006) y (2008-2009)” respecto a los resultados de la presente investigación

\begin{tabular}{|c|c|c|}
\hline Estudios & Estudio $1^{(17)}$ & Estudio $2^{(4)}$ \\
\hline $\begin{array}{l}\text { Estadística } \\
\text { de } \\
\text { Mortalidad } \\
\text { Materna }\end{array}$ & $\begin{array}{l}\text { Los departamentos con peores } \\
\text { Razones de Mortalidad Materna } \\
\text { Acumulada Estandarizada, } \\
\text { fueron Chocó, Guainía. Y los } \\
\text { tres departamentos que } \\
\text { registraron las RMMAe más } \\
\text { bajas fueron Casanare, Córdoba } \\
\text { y Quindío. }\end{array}$ & $\begin{array}{l}\text { En el año } 2018 \text { se presentó en el } \\
\text { departamento de Guainía una RMM } \\
\text { de } 328.9 \text { y en Antioquia, con una } \\
\text { RMM de } 64.98 \text { por cada } 100,000 \\
\text { nacidos vivos. }\end{array}$ \\
\hline $\begin{array}{c}\text { Causas de } \\
\text { Mortalidad } \\
\text { Materna }\end{array}$ & $\begin{array}{cl}\text { Las tres principales causas de } \\
\text { mortalidad materna fueron: } \\
-\quad \text { Trastornos } \\
& \text { hipertensivos en el } \\
& \text { embarazo. } \\
-\quad & \text { Otras condiciones } \\
& \text { obstétricas no } \\
& \text { clasificadas en } \\
& \text { otra parte. } \\
\text { - } & \text { Complicaciones } \\
& \text { del trabajo del } \\
& \text { parto y del parto. }\end{array}$ & 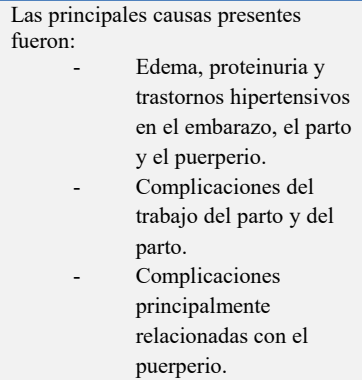 \\
\hline \multirow[t]{2}{*}{$\begin{array}{c}\text { Determinan } \\
\text { tes Sociales } \\
\text { en Salud }\end{array}$} & $\begin{array}{l}\text { Al analizar los datos de } \\
\text { población con necesidades } \\
\text { básicas insatisfechas - NBI, para } \\
\text { el año } 2005 \text { se encontró que los } \\
\text { departamentos con el NBI más } \\
\text { altos fueron: Chocó y Guainía. }\end{array}$ & $\begin{array}{l}\text { En la zona rural de Guainía la mujer } \\
\text { en gestación está expuesta a padecer } \\
\text { de malaria, dengue, leishmaniasis, } \\
\text { enfermedad de Chagas y fiebre } \\
\text { amarilla, además de estar a largas } \\
\text { distancias de los centros de salud con } \\
\text { niveles altos de atención. } \\
\text { Gran parte de estas mujeres tienen su } \\
\text { parto guiado por partera. Gran parte } \\
\text { de esta población cuenta con régimen } \\
\text { subsidiado. }\end{array}$ \\
\hline & $\begin{array}{l}\text { La Guajira y Vaupés. Y los de } \\
\text { proporción de NBI más bajos } \\
\text { fueron Bogotá, Quindío } \\
\text { Antioquia y Santander. }\end{array}$ & $\begin{array}{l}\text { Comparado con el departamento de } \\
\text { Antioquia, contando que el parto de } \\
\text { estas mujeres es atendido por } \\
\text { profesionales capacitados y en centros } \\
\text { médicos, tienen niveles económicos } \\
\text { superiores, viven en áreas urbanas, las } \\
\text { cuales tienen menor lejanía a los } \\
\text { centros de salud, y tienen mayores } \\
\text { niveles educativos. }\end{array}$ \\
\hline
\end{tabular}

Por último, Sandoval y Eslava, no incluyen políticas de salud pública en su revisión.

\section{CONCLUSIONES}

En el año 2018 la RMM es menor en el departamento de Antioquia dado que cuentan con mayor índice de nacidos vivos respecto a las defunciones maternas, con una RMM de 64.98 por 100,000 nacidos vivos, comparado a Guainía con una RMM de 328.95 por 100,000 nacidos vivos. 
En ese mismo año pudo evidenciarse que las principales causas de mortalidad materna en común en los dos departamentos fueron: "edema, proteinuria y trastornos hipertensivos en el embarazo, el parto y el puerperio (O10O16)", "Complicaciones del trabajo del parto y del parto (O60O75)" y "Complicaciones principalmente relacionadas con el puerperio (O85-O92)", lo cual es una base para realizar propuestas en salud pública que reduzcan el número de muertes maternas en estos departamentos.

Las políticas de Salud Pública en el 2018 no fueron totalmente efectivas, en estos territorios, en el departamento de Guainía sus actividades se centraron hacia la implementación de programas de planificación, procedimientos menores como canalizaciones o desparasitaciones y kits de insumos, pero dejó a un lado todo lo que comprende el tema de mortalidad materna, reflejado en su alza en la razón de mortalidad materna, cumpliendo de manera tolerable casi sobrepasando el límite de la meta propuesta para ese año; en el departamento de Antioquia se planteó políticas mucho más completas y manejables. Con esto se concluye que una posible renovación de las políticas en el departamento de Guainía en el futuro y una mejor implementación de las mismas en el departamento de Antioquia, podría ser determinante en la disminución de la razón de mortalidad materna. Así mismo, el departamento de Guainía se podría apoyar en las políticas tomadas en Antioquia y usarlas como ejemplo para implementar en su departamento.

Los determinantes sociales en salud influyen positiva o negativamente en la salud de la mujer gestante, las diferencias son notables, en la zona rural de Guainía la mujer en gestación está expuesta al contagio de enfermedades como malaria, dengue, leishmaniasis, enfermedad de Chagas y fiebre amarilla, localizadas en zonas apartadas de las entidades prestadoras de servicios de salud nivel de complejidad III y IV que corresponden a aquellas que cuentan con servicios de médicos y práctica de atención del parto en el lugar de vivienda por parteras, lo cual dificulta brindar atención y procedimientos médicos especializados o quirúrgicos ante complicaciones en el parto o puerperio. Gran parte de esta población cuenta con régimen subsidiado, lo que genera una menor calidad y disponibilidad de recursos en la atención en salud sin mencionar la baja cantidad de entidades prestadoras de servicios en salud y su acceso a ellas lo cual genera una repercusión en esta problemática de salud pública. Y la mayoría de estas mujeres presenta algún tipo de enfermedad periodontal (15), esto puede conllevar a alguna complicación en el embarazo. En el departamento de Antioquia, las condiciones en salud para gestantes son más favorables, los partos en su totalidad por profesionales capacitados y en centros médicos, con niveles económicos superiores en toda la población (15), viviendo un gran porcentaje de la población en áreas urbanas que disponen de centros de salud cercanos, y tienen mayores niveles educativos (15). Todo esto provoca una mayor incidencia en la mortalidad materna respecto al departamento de Guainía.

Conflicto de Interés: Los investigadores declaran no tener ningún conflicto de interés en la realización del estudio.

Agradecimientos: A Dios, la Fundación Universitaria Juan N. Corpas y los doctores Juan Carlos Correa Senior y María Luisa Latorre Castro, por ser nuestros mentores y guías respecto a su enseñanza en el tema de Salud Pública.

\section{REFERENCIAS}

1. Organización Panamericana de la Salud/ Organización Mundial de la Salud. Salud Materna [Internet]. SF. México [citado 01 de octubre de 2020]. Disponible en: https://www.paho.org/mex/index.php?option=com_content \&view $=$ article $\&$ id $=341$ :saludmaterna\&Itemid $=387 \#: \sim:$ text $=$ La\%20muerte\%20materna\%20es\%20definida,no\%20por\% 20causas $\% 20$ accidentales $\% 20$ o

2. Organización Mundial de la Salud. Objetivos de Desarrollo Sostenible: Metas [Internet]. 2020 [citado 01 de octubre 2020]. Disponible https://www.who.int/topics/sustainable-developmentgoals/targets/es/

3. Organización Panamericana de la Salud/ Organización Mundial de la Salud. "La mortalidad materna es inaceptable": Gina Tambini [Internet]. 2019. Colombia [citado 01 de octubre 2020]. Disponible en: https://www.paho.org/col/index.php?option=com_content\& view $=$ article $\&$ id $=3165$ :la-mortalidad-materna-esinaceptable-gina-tambini\&Itemid $=551$

4. SECRETARÍA DISTRITAL DE SALUD DE BOGOTÁ. Mortalidad materna [Internet]. SF [citado 27 de septiembre de 2020]. Disponible en: http://www.saludcapital.gov.co/sitios/VigilanciaSaludPubli ca/Protocolos $\% 20$ de $\% 20$ Vigilancia $\% 20$ en $\% 20$ Salud $\% 20 \mathrm{Pu}$ blica/Mortalidad\%20Materna.pdf

5. Organización Mundial de la Salud. Mortalidad materna [Internet]. 2019 [citado 27 de septiembre de 2020]. Disponible en: https://www.who.int/es/news-room/factsheets/detail/maternal-mortality

6. Instituto Nacional de Salud. Boletín Epidemiológico Semanal. Semana epidemiológica 52, 22 al 28 de diciembre de 2019 [Internet]. 2019 [citado 30 julio 2020]. Disponible en: https://www.ins.gov.co/buscadoreventos/BoletinEpidemiologico/2019_Boletin_epidemiolog ico_semana_52.pdf\#search=boletin $\% 20$ epidemiologico $\% 20$ semana $\% 2052 \% 202019$

7. DANE. Salud. Nacimientos y defunciones. Defunciones No fetales. [Internet]. SF [citado 18 septiembre 2020]. Disponible

https://www.dane.gov.co/index.php/estadisticas-portema/salud/nacimientos-y-defunciones/defunciones-nofetales

8. DANE. Salud. Nacimientos y defunciones. Nacimiento. [Internet]. SF [citado 18 septiembre 2020]. Disponible en: https://www.dane.gov.co/index.php/estadisticas-portema/salud/nacimientos-y-defunciones/defunciones-nofetales

9. Gobernación del departamento del Guainía. Informe Ejecutivo de Gestión. [Internet]. 2018 [citado 30 de septiembre de 2020]. Disponible en: https:/guainia.micolombiadigital.gov.co/sites/guainia/conte nt/files/000435/21716_informe-general-2018-terminadoultimo.pdf 
10. Gobernación del Guainía y Secretaría de Salud Departamental. Guainía avanza en la prevención de la mortalidad materna [Internet]. 2018. Nota: 024. Guainía: Gobernación del Guainía y Secretaría de Salud Departamental [citado 30 de septiembre de 2020]. Disponible https://guainia.micolombiadigital.gov.co/sites/guainia/conte nt/files/000081/4010_nota-codigo-roo-sexualidad.pdf

11. Dirección de salū pública, grupo de promoción y prevención a las direcciones territoriales departamentales y distritales del país. Lineamientos generales reducción de la mortalidad materna [Internet]. 2010. No. 13.340. Ministerio de Salud y Protección Social [citado 10 de octubre de 2020]. Disponible en: https://www.dssa.gov.co/index.php/programas-yproyectos/salud-publica/item/141-maternidad-segura

12. Instituto Nacional de Salud - Equipo de maternidad segura. Memorias de vigilancia epidemiológica de mortalidad materna basada en la web [Internet]. 2010. Ministerio de Salud y Protección Social [citado 10 de octubre de 2020]. Disponible

en: https://www.dssa.gov.co/index.php/programas-yproyectos/salud-publica/item/141-maternidad-segura

13. Organización Mundial de la Salud. Determinantes Sociales de la Salud. [Internet]. 2019 [Citado el 02 de octubre de 2020]. Disponible en: https://www.who.int/social_determinants/es/

14. Ministerio de Salud. Análisis de situación de salud de Colombia. [Internet]. 2013 [Citado el 02 de octubre de 2020]. Disponible en: https://semilleropacifico.uniandes.edu.co/images/document/ salud/Analisis-de-situacion-de-salud-segun-regiones-decolombia.pdf

15. Ministerio de Salud. Análisis de situación de salud con el modelo de los determinantes sociales de salud. [Internet]. 2018 [Citado el 02 de octubre de 2020]. Disponible en: http:/guadalupeantioquia.micolombiadigital.gov.co/sites/gu adalupeantioquia/content/files/000088/4373_asis_guadalup e_2018.pdf

16. Ministerio de Salud y Protección social. Protección social. Cifras de aseguramiento en salud. [Internet]. 2019 [citado 09 de septiembre de 2020]. Disponible en: https://www.minsalud.gov.co/proteccionsocial/Paginas/cifr as-aseguramiento-salud.aspx

17. Sandoval Y, Eslava J. Inequidades en mortalidad materna por departamentos en Colombia para los años (2000-2001), (2005-2006) y (2008-2009). [Internet]. 2013; 15(4): 529541. Rev. Salud pública [citado el 09 de septiembre de 2020]. Disponible

en: https://www.scielosp.org/article/rsap/2013.v15n4/579-591/ 